\title{
A NEW OPTICAL SYSTEM FOR SOLAR SOFT X-RAY SPECTROPHOTOMETRY
}

\author{
J. H. DIJKSTRA \\ Space Research Laboratory, Utrecht, The Netherlands \\ and \\ W. WERNER \\ Institute of Applied Physics, Delft, The Netherlands
}

\begin{abstract}
At the Institute of Applied Physics TNO-TH at Delft a strongly off-plane grating mounting has been developed for the soft X-ray wavelength region. In this mounting a parallel beam is incident on the grating surface nearly parallel to the grooves. We found herewith a remarkable improvement of the grating efficiency compared with the grazing incidence perpendicular to the grooves. The focal distance is nearly independent on the wavelength, so scanning of the spectrum can be provided by a simple rotation of the cylindrical grating around the central groove. The exit slit and the detector have fixed positions. The lightspot on the detector cathode does not move and has a moderate size; a standard detector can be used.

This mounting is applied in a rocket experiment to the measurement of the solar spectrum between $30 \AA$ and $80 \AA$. By means of a sieve plate collimator the solar beam is collimated to about one minute of arc resulting in a spectral resolving power of about $0.7 \AA$; with a better collimator it is possible to reduce this to about $0.2 \AA$.

The instrument has been flown on a Skylark rocket in November 1968. During this flight the field of view was pointed at the center of the solar disk. A second launch is in preparation.
\end{abstract}

